\title{
Técnicas asépticas en el cuidado enfermero a pacientes hospitalizados que requieren administración de fármacos y aspiración de secreciones del tracto respiratorio
}

\author{
Orellana-Carmona Miriam¹, Changa-Murga Rosa', Sullcapuma-Guillen Braulio', \\ Chávez-Cachay Esther ${ }^{2}$
}

\section{RESUMEN}

Las técnicas asépticas son muy importantes para reducir la posibilidad que los microorganismos entren al organismo durante la administración de fármacos y la aspiración de secreciones, a su vez, disminuyen el riesgo que los pacientes se infecten durante su estancia hospitalaria. Objetivo: determinar el cumplimiento de las técnicas asépticas en los procedimientos de administración de fármacos y aspiración de secreciones del tracto respiratorio por parte de los enfermeros durante el cuidado de pacientes con grado de dependencia III. Material y métodos: estudio descriptivo observacional realizado en los servicios de medicina de un hospital local, con una población de 78 enfermeros, a quienes se les aplicó una guía de observación. Resultados: el 51,3\% de las enfermeras no cumple con las técnicas asépticas en los procedimientos de administración vía oral y endovenosa de fármacos, y aspiración de secreciones del tracto respiratorio. Conclusiones: la mayoría de enfermeros de los servicios de medicina de un hospital local no cumple técnicas asépticas en la administración de fármacos vía oral y endovenosa y aspiración de secreciones del tracto respiratorio. Esto estaría incrementando el riesgo de los pacientes con grado dependencia III de adquirir infecciones intrahospitalarias. La técnica aséptica en aspiración de secreciones del tracto respiratorio fue la menos cumplida por las enfermeras en los servicios de medicina.

Palabras clave: cuidado, enfermeria, asépticas, infecciones, hospitalarias. Perú. (Fuente DeCs BIREME).

\section{Aseptic techniques in nursing care to hospitalized patients require drug delivery and aspiration of respiratory tract secretions}

\begin{abstract}
Aseptic techniques are very important to reduce the possibility of micro-organisms enter the body during the administration of drugs and the aspiration of secretions at the same time decrease the risk that patients from becoming infected during their hospital stay. Objetive: Determine the compliance of the aseptic techniques used in procedures for drug delivery and aspiration of secretions from the respiratory tract by nurses in the care of patients with dependence on III. Material and Methods: Descriptive observational study, made from eight services of Medicine of local hospital, with a population of 78 nurses, who was a guide of observation. Results: $51.3 \%$ of the nurse's meets do not meet aseptic techniques in all procedures in management procedures oral medications and intravenous drug and aspiration of respiratory tract secretions. Conclusion: The majority of nurses from the hospital medicine services meet aseptic techniques only when they perform one or two procedures; Administration of drugs via intravenous and oral and/or pick up secretions from the respiratory tract. This would increase the risk of patients with grade unit III, of acquiring nosocomial infections. The least accomplished technique is washing hands after the procedure. Aseptic technique in suctioning respiratory tract was the least accomplished by nurses in medical services.
\end{abstract}

Key words: Care, nursing, aseptic, infections, hospital. Peru. (Source DeCs BIREME).

Licenciada en Enfermería. Universidad Peruana Cayetano Heredia. Lima-Perú

Licenciada en Enfermería. Docente de la Facultad de Enfermería de la Universidad Peruana Cayetano Heredia. Lima-Perú 


\section{INTRODUCCIÓN}

La asepsia es la ausencia de microorganismos causantes de infección; para reducir las posibilidades de trasmisión de microorganismos se usa la técnica aséptica (1). La técnica aséptica consiste en un conjunto de procedimientos y actividades destinados a disminuir al mínimo las posibilidades de contaminación microbiana de pacientes y equipos. Esta conducta, junto a la introducción de los conceptos de antisepsia, desinfección y esterilización, han logrado un impacto importante de la prevención de infecciones intrahospitalarias (2).

La capacidad de un microorganismo de causar una infección depende de varios factores, uno de los más importantes es la susceptibilidad del huésped, que se ve afectada por la edad, la herencia, el nivel de estrés, el estado nutricional, el tratamiento médico actual y las enfermedades previas (3). El cumplimiento de las técnicas asépticas en el cuidado del paciente hospitalizado evita que se creen condiciones que lo predispongan a contraer una infección intrahospitalaria (3).

En el área hospitalaria, la labor del personal de enfermería está centrada en el cuidado integral del ser humano a fin de lograr y/o mantener un estado de salud óptimo, por lo que se debe enfatizar las medidas de prevención de enfermedades y limitación de riesgos. Los procedimientos invasivos y la alteración de las defensas inmunitarias son los principales factores contribuyentes a las infecciones nosocomiales. El Centro de Control de Enfermedades (CDC) de Atlanta, en el año 2005, reporta que solo en EE. UU. más de 80000 pacientes mueren anualmente debido a una infección intrahospitalaria (IIH). Estas originan, además de un prolongado tiempo de hospitalización, una carga económica de unos 5000 millones de dólares al año. Un tercio de estas muertes y una fracción mayor de los gastos, podrían evitarse con programas de control de infecciones, y con el cumplimiento de normas preventivas, entre ellas las técnicas asépticas (4).

El costo económico en salud de los hogares incluye el pago de bienes y servicios para la prevención, detección, tratamiento y rehabilitación, es decir, los componentes que constituyen el costo directo de la atención. Las consecuencias económicas de las infecciones nosocomiales se traducen en una elevada carga de tratamiento para los hospitales a consecuencia del uso de recursos materiales para el control y tratamiento (costo directo). Así mismo, ellos representan una carga monetaria para el paciente y su familia (costo indirecto) (4).
Las infecciones contraídas en el hospital generalmente son causadas por gérmenes multirresistentes que solo responden a las antibióticos más caros, lo cual incrementa los costos. Por ello, se debe cumplir con las técnicas asépticas evitando posibles infecciones oportunistas (5). Una encuesta de prevalencia realizada en el año 2006, bajo los auspicios de la Organización Mundial de la Salud (OMS), en 55 hospitales de catorce países representativos de cuatro regiones de la OMS (Europa, el Mediterráneo Oriental, el Asia Sudoriental y el Pacífico Occidental) mostró que un promedio de $8,7 \%$ de los pacientes hospitalizados presentaban infecciones nosocomiales (5).

En un momento dado, más de 1,4 millones de personas alrededor del mundo sufren complicaciones por infecciones contraídas en el hospital, esto puede estar relacionado con la falta de asepsia por parte del personal de salud (5).

En Chile se notifican aproximadamente 30000 infecciones intrahospitalarias (IIH) al año, siendo en el año 2006 la tasa de incidencia global de $10 \%$ y se estima, con estudios de prevalencia, que la cifra real es el doble. Uno de los factores de riesgo de IIH está relacionado con la atención hospitalaria, que es donde existe la mayor posibilidad de intervenir, dado que es la responsabilidad directa del personal de salud. Existen diversas medidas dependientes del tipo de infección, pero las comunes y más importantes tienen que ver con el uso de técnica aséptica cuando se requiere $(6,7)$.

Según información del Plan Operativo Anual 2011 del Hospital Nacional Arzobispo Loayza, en el Perú se presentan 50000 infecciones intrahospitalarias anuales, lo que está por encima de estándares internacionales relacionadas al uso de la asepsia en la atención del paciente hospitalizados (7). La oficina de Epidemiología, en el año 2011, determinó que en los servicios de medicina, el $77 \%$ no utiliza barreras protectoras, que es uno de los principios de asepsia (8).

La motivación para realizar la presente investigación surge de la observación realizada en nuestras prácticas profesionales en los servicios de medicina de un hospital local, donde se hospitalizan pacientes adultos con diferentes diagnósticos clínicos y se destacan los pacientes con grado de dependencia III, cuyo cuidado necesita del cumplimiento permanente de técnicas asépticas por parte del personal de enfermería en los procedimientos que ellos requieren durante su estadía hospitalaria.

La observación al personal de enfermería en los servicios mencionados permitió identificar que varios de ellos no 
realizaban el lavado de manos adecuadamente, ni con la frecuencia que debería darse. Asimismo, realizaban los procedimientos de administración de fármacos y aspiración de secreciones sin el uso correcto de guantes y delantal limpio, ni mascarilla en caso de aspiración de secreciones.

La situación mencionada anteriormente llevó a formular la siguiente pregunta:

¿Cuáles son las técnicas asépticas cumplidas por los enfermeros en el cuidado de pacientes con grado dependencia III que requieren procedimientos de administración de fármacos y aspiración de secreciones del tracto respiratorio en los servicios de medicina de un hospital local de mayo a junio de 2012 ?

La investigación tuvo el propósito de indagar sobre el cumplimiento de las técnicas asépticas por parte de los enfermeros en los procedimientos de la administración de fármacos y aspiración de secreciones al paciente hospitalizado con grado de dependencia III en los servicios de medicina de un hospital local.

\section{MATERIAL Y MÉTODOS}

\subsection{Tipo de estudio}

Estudio cuantitativo y descriptivo observacional, que permite determinar el cumplimiento de técnicas asépticas por parte del personal de enfermería que realizó administración de fármacos (V.O. y E.V.) y aspiración de secreciones a los pacientes con grado de dependencia III hospitalizados en los servicios de medicina de un hospital local.

\section{2 Área de estudio}

El estudio de investigación se desarrolló en los servicios de hospitalización de medicina en un hospital local, en dichos servicios mensualmente atienden aproximadamente 215 pacientes, entre 18 a 80 años con diferentes síndromes clínicos. Cada servicio cuenta con nueve a diez profesionales de Enfermería, quienes brindan cuidado a pacientes con grado de dependencia III; dichos pacientes no participan en su cuidado; son crónicos inestables; requieren de valoración continua y monitoreo; tienen alteración significativa de signos vitales; disminución del estado de conciencia; con medicación parenteral permanente, y tratamiento frecuente. Puede o no tener usos de aparatos especiales de soporte de la vida, por lo cual el personal de Enfermería realiza diversos procedimientos, siendo los más frecuentes la administración de medicamentos por diferentes vías y la aspiración de secreciones del tracto respiratorio.

\subsection{Población}

La población estuvo conformada por 78 licenciados en Enfermería, que laboran en los servicios de medicina de un hospital local; cumplen turnos de seis horas (mañana a tarde), y de doce horas diurnas y nocturnas respectivamente.

La población del estudio reunió los siguientes criterios:

Criterios de inclusión

* Licenciado o licenciada en Enfermería que labore permanentemente en el área asistencial en los servicios de medicina de un hospital local.

\section{Criterios de exclusión}

* Licenciado o licenciada en Enfermería en servicio de retén, o que cubre turnos de vacaciones y licencias.

\subsection{Técnica e instrumento de recolección de datos}

La técnica utilizada para la recolección de datos fue la observación; como instrumento se empleó una guía de observación que estuvo compuesta por tres dimensiones: técnicas asépticas en la administración de fármacos por vía oral (V.O.), vía endovenosa (E.V.) y la aspiración de secreciones del tracto respiratorio.

La guía de observación fue sometida a juicio de expertos, constituidos por licenciadas de Enfermería, algunas encargadas de la jefatura de los servicios de medicina y otras que laboran en el Departamento de Epidemiología del mismo hospital (Anexo 5). Terminado el proceso de validación del instrumento, se llevó a cabo la prueba piloto con diez licenciados en Enfermería de un servicio de hospitalización de medicina de un hospital local y que presentaron los mismos criterios de inclusión señalados. Con la guía de observación se evaluó tres dimensiones de la variable de estudio; siete ítems referidos a la administración del tratamiento por vía oral; dieciseis referidos a la administración de tratamiento E.V y nueve referidos a la aspiración de secreciones del tracto respiratorio en los pacientes con grado de dependencia III.

Todo/a enfermero/a fue observado/a en cada una de las tres dimensiones; se obtuvo una puntuación de «1» cada vez que realizó el ítem correspondiente, $\mathrm{y} « 0$ » cuando no lo realizó. Cada enfermero fue observado en tres momentos diferentes (mañana, tarde y noche); por la mañana de $8.00 \mathrm{~h} 12.00 \mathrm{~h}$; por la tarde de $15.00 \mathrm{~h}$ a $18.30 \mathrm{~h}$ y en el turno noche de 20.00 h a 23.30 h. En un día, turno de doce horas, el enfermero era observado dos veces, turno mañana y tarde, al día siguiente, cuando le correspondía turno noche, se completaba con la tercera observación, para 
cada enfermero, la observación correspondiente a cada turno fue con el primer paciente que cumplía con las características mencionadas; es decir, un paciente con grado de dependencia III, al cual se le realizaba las tres dimensiones. Para decir que dicho enfermero cumple con las tres dimensiones se empleó la siguiente escala de clasificación:

\begin{tabular}{lrl}
\hline & Escala & Clasificación \\
\hline Por vía oral & 0 a 12 & No cumple \\
& $>12$ & Cumple \\
Por vía endovenosa & 0 a32 & No cumple \\
& $>32$ & Cumple \\
Aspiración de & 0 a 20 & No cumple \\
secreciones & $>20$ & Cumple \\
\hline
\end{tabular}

Una vez aplicada la guía a la población de estudio se calculó las medias aritméticas (X) para cada aspecto (A) y el total; estos promedios fueron los puntos de cohorte para clasificar a los enfermeros en dos categorías (Anexo 4).

\subsection{Plan de recolección de datos}

La recolección de datos se realizó durante dos meses, diariamente durante el horario de trabajo previa coordinación con la jefatura de Enfermería. Se realizó una observación individual y neutral a los enfermeros de los servicios de hospitalización de medicina en tres momentos diferentes durante su jornada laboral (mañana, tarde y noche). La prueba piloto permitió saber que los momentos para poder observar las técnicas asépticas que cumplieron los enfermeros en la administración de fármacos por vía oral, vía endovenoso y en la aspiración de secreciones eran en determinadas horas, es por ello que en la ejecución de la recolección de datos cada uno de los investigadores asistió diariamente.

\subsection{Plan de tabulación y análisis de datos}

Los datos fueron analizados en el paquete estadístico SPSS (StatysticalPackage Social Sciencies) for Windows 15.0. Para determinar el cumplimiento de las técnicas asépticas en los procedimientos de administración de fármacos (por vía oral y endovenoso y aspiración de secreciones del tracto respiratorio, se obtuvieron tablas de distribución de frecuencias según las dimensiones y escala de clasificación del instrumento (cumple, no cumple). Para lograr dicho objetivo se obtuvieron tablas univariadas expresadas en números y porcentajes relacionados al cumplimiento de las técnicas asépticas por vía oral, endovenosa y aspiración de secreciones del tracto respiratorio en el cuidado de pacientes con grado de dependencia III. Los resultados se presentaron en cuatro gráficos.

\section{RESULTADOS}

Gráfico 1. Cumplimiento de las técnicas asépticas en los procedimientos de administración de fármacos y aspiración de secreciones del tracto respiratorio

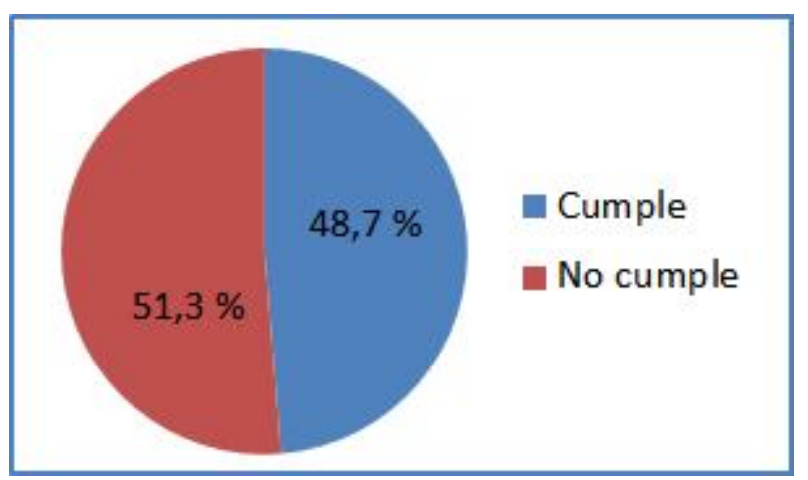

El 51,3\% del personal profesional de enfermería no cumple con las técnicas asépticas en los procedimientos de administración vía oral y endovenoso de fármacos y aspiración de secreciones del tracto respiratorio.

\section{Gráfico 2. Cumplimiento de las técnicas asépticas en la administración de fármacos por vía oral}

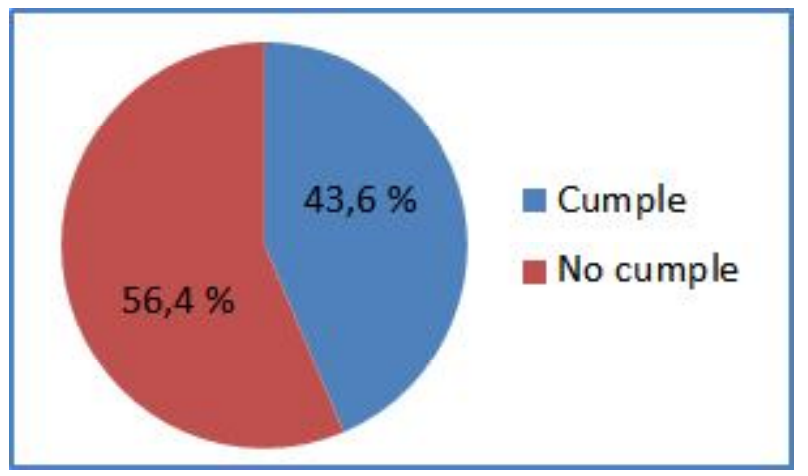

El 56,4\% del personal profesional de enfermería no cumple con las técnicas asépticas en el procedimiento de administración de fármacos vía oral.

\section{Gráfico 3. Cumplimiento de las técnicas asépticas en la administración de fármacos por vía endovenosa}

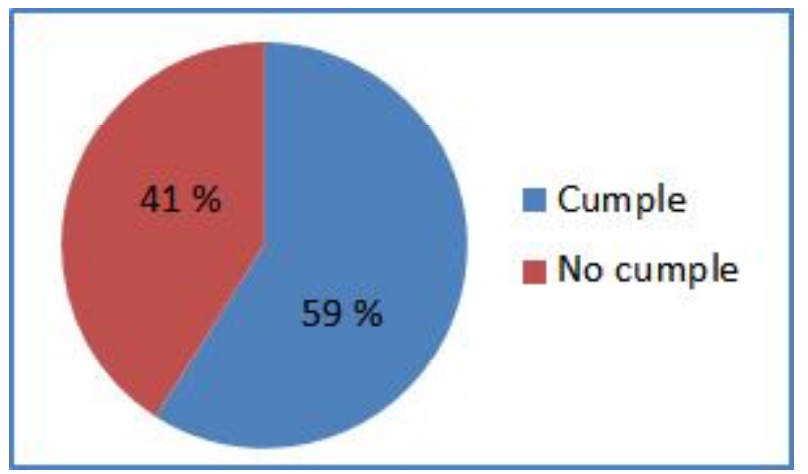


El 59\% del personal profesional de enfermería cumple con las técnicas asépticas en el procedimiento de administración de fármacos por vía endovenosa.

\section{Gráfico 4. Cumplimiento de las técnicas asépticas en la aspiración de secreciones del tracto respiratorio}

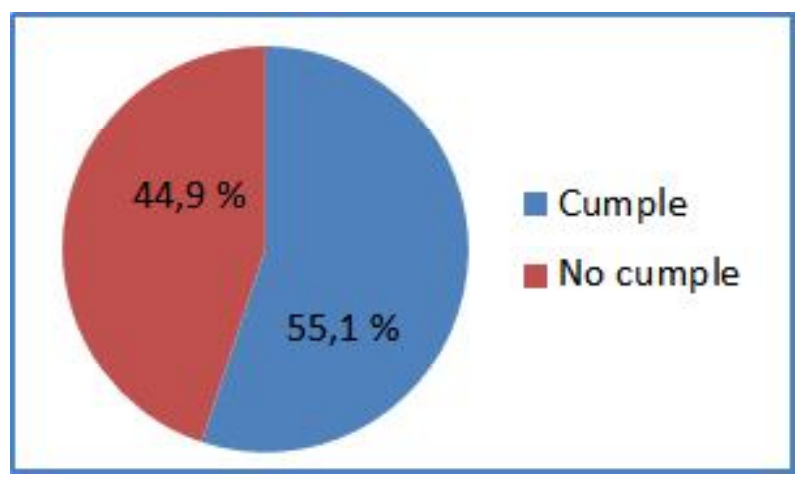

El 55,1\% del personal profesional de enfermería cumple con las técnicas asépticas en el procedimiento de aspiración de secreciones del tracto respiratorio.

\section{DISCUSIÓN}

El cuidado de enfermería a los pacientes se desarrolla con conocimientos, habilidades y valores. El cuidado no solo requiere que el profesional en Enfermería sea científico, académico y clínico, sino que, a la vez, debe de ser un agente de humanización (9); demostrar responsabilidad de técnicas asépticas durante la ejecución de procedimientos que requiere todo paciente hospitalizado. Las técnicas asépticas se refieren a las prácticas seguidas inmediatas antes o durante un procedimiento clínico, basadas en los principios científicos que se utilizan para destruir o prevenir la transmisión de microorganismos patógenos y no patógenos; al mismo tiempo de reducir el riesgo de infección en el paciente (10).

Las técnicas asépticas que se emplean tienen la finalidad de disminuir el riesgo de transmisión de microorganismos oportunistas de cualquier fuente hospitalaria; dichas técnicas deben ser utilizadas antes de realizar cualquier procedimiento clínico y aplicadas en el manejo de todos los pacientes hospitalizados con o sin diagnóstico de infección desde su ingreso hasta su alta, de esta manera se preserva la seguridad del paciente, definida por la Organización Mundial de la Salud (OMS) como la ausencia o reducción, a un nivel mínimo aceptable, de riesgo de sufrir un daño innecesario en el curso de la atención sanitaria $(11,12)$.

En los servicios de medicina se pudo observar que el 51,3\% del personal profesional de enfermería no cumple con las técnicas asépticas en los procedimientos de administración vía oral y endovenoso de fármacos y aspiración de secreciones del tracto respiratorio (Gráfico 1).

Los cambios del estado de salud también pueden alterar el delicado equilibrio que existe entre el ser humano y los microorganismos heterogéneos que subsisten en su interior. Por ejemplo, la hospitalización de un paciente puede hacer que microorganismos normalmente no virulentos de la bucofaringe sean sustituidos por bacilos gramnegativos (Klebsiella, Pseudomona) que invaden los pulmones y producen neumonía. Cuando el sistema inmunitario del paciente es defectuoso, el sujeto es más vulnerable a la enfermedad producida por patógenos oportunistas (13). Es por ello que el uso de técnicas asépticas durante los procedimientos de enfermería reducen el riesgo del paciente de contraer infecciones oportunistas durante su estancia hospitalaria, obteniendo una pronta restauración del estado de salud ya que se minimizan los factores predisponentes de infección. La responsabilidad, efectividad, eficiencia y orientación profesional de la enfermera son aspectos importantes que contribuyen a la seguridad del paciente, dimensión fundamental de la calidad asistencial (14).

Cuando las técnicas asépticas no son empleadas por el profesional de Enfermería durante la ejecución de procedimientos, se aumenta el riesgo de adquirir una infección intrahospitalaria; es decir, se agrega un factor que puede desarrollar una enfermedad diferente a la del motivo de ingreso, incrementando la estancia hospitalaria y, con ello, los costos, creándose un problema económico indirecto para el paciente y su entorno familiar (14).

La mayoría de infecciones durante la hospitalización de los pacientes se debe a patógenos oportunistas; es decir, unos microorganismos que forman parte de la microflora normal del paciente (Staphylococcus aureus, Escherichia coli, Candida albicans). En condiciones normales estos microorganismos no producen enfermedad, pero sí la provocan cuando son introducidos en localizaciones no protegidas (el torrente sanguíneo o los tejidos) (15). Las bacterias oportunistas aprovechan las condiciones preexistentes, que la vulnerabilidad del paciente potencia, para desarrollarse y originar una enfermedad de mayor gravedad (15). En los servicios de hospitalización de medicina, los pacientes, por lo general, son de grado de dependencia II y III, que cursan con enfermedades crónicas, las cuales pueden ocasionar inmunosupresión. Uno de los factores que contribuyen a la aparición de las infecciones hospitalarias es el huésped inmunosuprimido, es decir, un paciente cuyas defensas normales han disminui- 
do debido a una enfermedad. Por ello, es importante que el profesional de Enfermería cumpla con las técnicas asépticas en todos los procedimientos evitando así la aparición de infecciones intrahospitalarias en pacientes con grado de dependencia III.

Los microorganismos bacterianos que con más frecuencia producen infecciones intrahospitalarias son Echericha coli, Pseudomona aeruginosa, Staphyloccoccus aureos, Klesbsiella pneumoniae y microorganismos fúngicos como Candida albicans. De las especies mencionas la más aislada es la $P$. aeruginosa, asociada con la contaminación de fuentes comunes como el agua, antisépticos y equipos médicos. Para la prevención de infecciones nosocomiales se tiene como medida básica el lavado de manos antes y después del procedimiento, dicha medida reduce el $50 \%$ del riesgo de adquirir una infección; el uso de guantes estériles también es importante (17). Las manos del personal son los vehículos comunes para la propagación de los microorganismos. Para disminuir el riesgo de infecciones cruzadas, se debe emplear el lavado de manos, el uso concertado de precauciones de barrera y la limpieza completa del ambiente de los pacientes18. Uno de los principios de la técnica aséptica es el lavado de manos, pues es la medida básica más importante $\mathrm{y}$, a la vez, más simple, para prevenir las infecciones intrahospitalarias (19).

Para el lavado de manos se utilizan diferentes productos, entre ellos tenemos a los alcoholes, que presentan una actividad excelente frente a todos los grupos de microorganismos, con excepción de las esporas, y no son tóxicos; estos compuestos no poseen actividad residual y son inactivados por la materia orgánica. Se tiene que tener en cuenta que cada nuevo lavado con alcohol, disminuye su efectividad, por lo que se recomienda que de cada 4 a 5 usos de alcohol se realice un lavado de manos.

La clorhexidina, jabón antiséptico empleado en el lavado de manos, presenta una potente actividad antimicrobiana. Tiene un importante efecto acumulativo, su acción antimicrobiana aumenta con el uso periódico y su actividad no se afecta por la presencia de sangre u otras sustancias orgánicas.

Según lo observado, el personal profesional de enfermería no realizó una adecuada técnica de lavado de manos, ni tampoco la aplicó al momento de administrar el tratamiento farmacológico (VO, EV) entre paciente y paciente; pero sí usó alcohol gel; sin embargo, se recalca que el uso del alcohol gel no sustituye el lavado de manos.

Lenin obtuvo que del $100 \%$ de los aéreas evaluadas (emer- gencia, hospitalización, laboratorio y consultorios externos), con respecto a la técnica correcta del lavado de manos, se encontró que no se realiza una correcta técnica del lavado de manos clínico y fricción cuando emplean un producto a base de alcohol. El área de hospitalización presenta un 50\% de incumplimiento de la técnica del lavado de manos; mientras que en nuestro estudio no se cumplió con el lavado de manos después del procedimiento de vía oral y endovenosa (20).

Por otro lado, Ponce G. et al., muestran resultados donde el $86 \%$ de los sujetos observados llevan a cabo en menos de veinte segundos el lavado de manos, y el $10 \%$ lo omitió, lo que muestra que los proveedores del cuidado ya sea médicos, enfermeras o estudiantes no lo ubican como prioridad para prevenir y controlar las infecciones intrahospitalarias (21). Becerra et al., demostraron que el 95,31\% del personal de enfermería realiza el lavado de manos antes de cada procedimiento; 97,66\% lo realiza después de cada procedimiento y el $89,06 \%$ aplica las técnicas adecuadas al momento de lavarse las manos. Además, un 99,22\% hace uso correcto de guantes al momento de preparar el tratamiento farmacológico (22).

Se obtuvo que el $43,6 \%$ de las enfermeras cumplen con las técnicas asépticas en el procedimiento de administración de fármacos vía oral. (Gráfico 2). Dentro de esta dimensión se recalca que la técnica aséptica menos usada fue el lavado de manos. La medida universal es el lavado de manos, por lo que la enfermera debe insistir y persistir en su práctica diaria a fin de contribuir con la disminución de la incidencia y/o prevención de infecciones intrahospitalarias, todo lo que la enfermera conoce lo tiene que aplicar para dar seguridad al paciente; no obstante, debe de emplearse antes y después de la atención directa de pacientes (23). Si bien es cierto que la técnica aséptica que en mayor porcentaje no cumplió el personal profesional de enfermería fue el lavado de manos; también se observó que existen otras prácticas que no hacen con frecuencia, entre ellas se encuentran las técnicas de asepsia, desinfección y esterilización del equipo y materiales antes de administrar tratamiento vía oral y endovenoso y aspiración de secreciones. Estas deficiencias en la aplicación de las técnicas asépticas representan resultados altamente riesgosos para el paciente; este cuidado inefectivo está expone al daño y esa no es la finalidad por la cual un profesional de enfermería forme parte fundamental del equipo de salud en la atención integral del paciente.

El cumplimiento de la asepsia y desinfección en caso de la administración de fármacos por vía endovenosa dismi- 
nuye la posibilidad del paciente de presentar flebitis. El $41 \%$ del personal profesional de enfermería no cumple con las técnicas asépticas en el procedimiento de administración de fármacos por vía endovenosa, lo que aumentaría el riesgo de flebitis en los pacientes del servicio de medicina. La flebitis es catalogada como una infección nosocomial; es un problema que el profesional de Enfermería debe evitar en su trabajo cotidiano ya que está ligada en gran medida a la calidad del mismo, es un indicador hospitalario de eficiencia, eficacia y efectividad en la atención de los pacientes con tratamiento a través de una vía intravenosa periférica permanente; por ello, es importante conocer objetivamente sus causas (24).

La flebitis relacionada con la contaminación microbiana de la infusión es menos frecuente que la asociada con el catéter, la contaminación es posible durante la preparación, manipulación del frasco y/o de los dispositivos conectados a este, como equipos de venoclisis o extensiones. Para la prevención de infecciones del torrente sanguíneo se debe dar mantención de circuito cerrado en la terapia intravascular y reducción de la manipulación al mínimo indispensable; además de utilizar una técnica aséptica en el procedimiento, los catéteres deben cambiarse en un periodo no mayor a $72 \mathrm{~h}$. Por ello, se debe garantizar el correcto cumplimiento de técnicas asépticas en la administración de fármacos por vía endovenosa, para mantener la seguridad en el cuidado del paciente evitando infecciones intrahospitalarias.

Las infecciones hematógenas ligadas al uso de un catéter derivan en gran parte de la microflora cutánea del sitio de inserción, donde los microorganismos emigran por vía intraluminal hasta la punta del catéter, casi siempre durante la primera semana después de su inserción. Los microorganismos patógenos más comunes en las bacteriemias ligadas a un instrumento vascular son Estafilococo coagulasa negativo, S. Aureus, Enterococo, bacilos nosocomiales gramnegativos y Candida (25).

Por otro lado, las infecciones relacionadas con el uso del catéter endovenoso producen un aumento de los costos directos de atención, que repercuten en la institución hospitalaria en dos aspectos importantes: elevación del número de días de estancia hospitalaria y gastos secundarios en tratamiento antimicrobiano, las pruebas diagnósticas como radiografías y exámenes de laboratorio, poniendo en tela de juicio las acciones de intervención en la calidad de atención de alta especialidad (26). Los catéteres y aparatos intravasculares constituyen una causa común de infección local, pues originan hasta $50 \%$ de las bacteriemias intrahospitalarias; por ello, se debe emplear una técnica aséptica para introducir dichos catéteres. La Organización Mundial de la Salud menciona que un $40 \%$ de las inyecciones se administran con jeringas y agujas reutilizadas sin esterilización previa y, en algunos países, esa proporción asciende hasta un 70\% (27). Según Segovario y Viscaya el 88,99\% utiliza medidas de asepsia y antisepsia; el $100 \%$ utiliza guantes limpios y realiza lavado de manos antes y después de la preparación de medicamentos endovenosos (28).

Barrera y Cieza hallaron que en el 56,5\% de pacientes, el catéter periférico permaneció $48 \mathrm{~h}$ en la vena y, en ese mismo tiempo, comenzó a aparecer el mayor número de flebitis $(17,39 \%)$, atribuyendo una relación inversa entre la aparición de flebitis y el lavado de manos. Alayza et al. mencionan que la flebitis pospunción constituye un problema que atañe directamente al profesional de Enfermería; al parecer, la sintomatología de menos de 24 h puede estar relacionada con la asepsia en la preparación, aplicación, administración y manipulación de la vía (29).

Rojas I., evaluó 112 pacientes hospitalizados en los servicios de medicina, cirugía, quemados, UCIG y otros, de ellos, el 62,5\% (70) presentó infección intrahospitalaria por Pseudomona aeruginosa. De estos casos el factor de riesgo extrínseco más frecuente fue el catéter endovenoso periférico (52 casos) (30). La desinfección y esterilización de materiales de uso médico son medidas comprobadas de prevención de infecciones. La mayoría de las acciones médicas y de enfermería que se ejecutan en la atención de los pacientes requieren que los elementos utilizados deban necesariamente ser esterilizados o desinfectados. Rojas también menciona que debido a que es innecesario esterilizar todos los materiales en el Hospital Nacional Arzobispo Loayza, se identifica si el material debe ser limpiado, desinfectado o esterilizado, de acuerdo a la clasificación del material, por Earle H. Spaulding, hace casi 30 años, donde describe tres tipos de materiales críticos, semicríticos y no críticos, los cuales requieren esterilización, desinfección de alto nivel y desinfección de bajo nivel, respectivamente. El 44,9\% de las enfermeras no cumple con las técnicas asépticas en el procedimiento de aspiración de secreciones del tracto respiratorio.

Dentro de las técnicas asépticas en el procedimiento de aspiración de secreciones se observó que las enfermeras no utilizaron un frasco limpio para almacenamiento de secreción aspirada ni emplearon una sonda estéril para cada zona de aspiración; siendo estas últimas técnicas asépticas también relevantes, ya que los frascos colectores de cada paciente deben ser vaciados y lavados las veces que sea necesario, y entre paciente y paciente, evi- 
tando la contaminación microbiana durante la aspiración de secreciones del tracto respiratorio (31). Tales prácticas intervienen directamente en la cadena epidemiológica para la transmisión y colonización de objetos inertes o directamente en el paciente, considerando que la transición de la flora comunitaria a la nosocomial es un evento factible en dicha cadena epidemiológica con lo que se previene la transmisión de infecciones cruzadas dentro de la estancia hospitalaria del paciente, está infección aparece como consecuencia de la interacción agente, huésped y medio donde muchas circunstancias influyen en contra del huésped, como la inmunodeficiencia que puede presentarse por estrés (hospitalización, enfermedad, procedimientos invasivos y tratamientos inmunosupresores), motivo por el cual se debe guardar un estricto control en su manejo (32).

Los profesionales de Enfermería deben lavarse las manos antes y después de una asistencia de cualquier tipo, hacer uso de una técnica aséptica estricta cuando se cambian las soluciones y tubos siguiendo las normas hospitalarias, además de comprobar la fecha de caducidad y la integridad del empaquetado de todo el material estéril. Es importante resaltar que la forma más frecuente de trasmisión es la infección cruzada (propagación indirecta de los patógenos de un paciente a otro a través de las manos mal lavadas del personal del hospital) o bien la autoinoculación al realizar la aspiración de la flora bucofaríngea al pulmón a lo largo de una sonda endotraqueal. Motivo por el cual, se debe realizar un lavado de manos antes y después de algún procedimiento y utilizar el equipo de protección necesario para evitar de trasmisión microorganismos patógenos (33).

Las manos con guantes estériles mantienen la esterilidad de la sonda de aspiración. Aunque las vías respiratorias altas (orofaringe y nasofaringe) no están estériles, se recomienda usar una técnica estéril en todas las aspiraciones para evitar la introducción de gérmenes patógenos, dando lugar a una colonización transitoria en las vías respiratorias bajas como la laringe, la tráquea y los bronquíolos que suelen ser estériles. Por lo general, la enfermedad aguda de las vías respiratorias inferiores se debe a bacterias orales más virulentas (S. pneumoniae, S. aureus y Klebsiella) (34).

Los guantes reducen la probabilidad de que el personal trasmita sus propios microorganismos endógenos a los sujetos que reciben su asistencia o trasmitan microorganismos de un paciente a otro. En todas las situaciones, los guantes se cambian entre los contactos con los pacientes, y las manos se lavan cada vez que se reti- ran los guantes (35). Se debe tener en cuenta que se utilizará una sonda por cada aspiración (boca y tráquea). $\mathrm{Al}$ finalizar la aspiración de secreciones se debe lavar con abundante agua estéril la goma de aspiración para facilitar el drenaje completo de secreciones hacia el frasco y mantenga protegida la conexión luego de realizada la aspiración. Para reducir la frecuencia de IIH, como por ejemplo la neumonía intrahospitalaria, se debe mantener una asepsia rigurosa del equipo utilizado, es decir, desinfectar o esterilizar todos los componentes que se utilizan. Además, se debe tener en cuenta que en pacientes con alteración del nivel de conciencia aumenta la posibilidad de aspiración del contenido bucofaríngeo en la parte inferior del aparato respiratorio, por lo cual es de suma importancia el uso de las técnicas asépticas en la aspiración de secreciones en pacientes con grado de dependencia III. López Schwartz refiere que existe un porcentaje considerable $(53,3$ y $60 \%)$ de enfermeras que aplican de manera inadecuada la aspiración de secreciones a pacientes traqueostomizados antes y después del procedimiento, relacionado a que: no prepara equipos y materiales para boca y traqueostomía, no retira joyas en el momento de lavarse las manos y no se coloca mandilón y mascarilla. Asimismo, García M, et al. muestran que el $60 \%$ de enfermeras del Hospital de Clínicas e Instituto Nacional de Tórax no utilizan los equipo de protección durante la aspiración de secreciones, mientras que en el presente estudio se obtuvo que el 44,9\% no hace uso de la vestimenta estéril como colocación mascarilla, gorro mandil y guantes estériles (36). Cubas obtuvo que el $100 \%$, de 21 enfermeras no verifica campo estéril ni se coloca el mandilón al realizar aspiración de secreciones; sin embargo, dicho porcentaje sí realiza el lavado de manos, se coloca mascarilla y guantes estériles. Por otro lado, el $81 \%$ (17) y $100 \%$ (21) se lavan las manos antes y después del procedimiento, respectivamente. A diferencia que la población de estudio, no cumplió con el uso de la vestimenta estéril y limpia, lo cual pone en riesgo su salud, al personal y la del propio paciente (37).

\section{REFERENCIAS BIBLIOGRÁFICAS}

1. Sciencie Communication Network. Aseptic Techniques. [Internet] 2010 abril 15. [citado $18 \mathrm{Feb}$ 2012]. Disponible en: http://www.ems.org.eg/esic_ home/data/giued_part1/Aseptic_Techniques.pdf.

2. Lemone P, Burke K. Enfermería Médico Quirúrgico. 4. a ed. Madrid: Pearson Prentice Hall; 2009. Vol. I.

3. Barnaul A, Jo S, Kozier B. Fundamentos de Enfermería conceptos, procesos y práctica. 8. a ed. México: GlenoraErb; 2008. 
4. Ministerio de Salud. Guía técnica para la evaluación interna de la vigilancia, prevención y control de las infecciones intrahospitalarias RM 523-2007/MINSA [Internet] 2007 junio 10 [citado 01 May 2011]. Disponible en: http://bvs.minsa.gob.pe/local/MINSA/ 1219_DGSP280.pdf

5. Arévalo H, Cruz R, Palomino F, Fernández F, Guzmán E, Melgar R. Aplicación de un programa de control de infecciones intrahospitalarias en establecimientos de salud de la región San Martín. Rev. Perú Med. 2005 Noviembre; 20(2):214.

6. Ajenjo C. Infecciones intrahospitalarias: Conceptos actuales de prevención y control. Revista Chilena de Urología 2006 Mayo; 71(2):95.

7. Hospital Nacional Arzobispo Loayza. Plan Operativo Anual 2011. [internet].2010 febrero. [citado 16 Feb 2012] Disponible en: http://www.hospitalloayza.gob. pe/transparencia/files/TRA_b763c0d1491f6db_H NAL.pdf

8. Oficina de Epidemiologia y Estadística del Hospital Nacional Arzobispo Loayza. Epidemiologia y estadística de los servicios de medicina del Hospital Nacional Arzobispo Loayza; 2010.

9. Potter P. Fundamentos de enfermería. 5.a ed. Madrid: Harcourt Brace; 2001.

10. Engenderhealth. De prevención de infecciones. Manual de referencia para proveedores de servicios de salud. [Internet].2001 enero 7. [citado 29 Agos 2012]. Disponible en: http://www.fernocas.com/ tecnicas_procedimientos/tecnicas/quirofanos.pdf.

11. Organización Mundial de la Salud. Curso sobre seguridad del paciente. Proyecto Bacteriemia Zero. [Internet] 2008 agosto 20 [citado 29 Agos 2012]. Disponible en: http://hws.vhebron.net/formacionBZero/docs/CURSO_PSI_BZ.pdf.

12. Rivera R, Castillo G, Astete M. Eficacia de un programa de capacitación en medidas básicas de prevención de Infecciones Intrahospitalarias. Rev. Perú. Med. [Internet]. 2005 marzo 18. [citado 25 Jul 2012].Vol. 22 (N. ${ }^{\circ}$ 2/p.88-95). Disponible en: http:// www.scielo.org.pe/scielo.php?script $=$ sciart text\&pid=S 1726-46342005000200002\&Ing=es $\& n r m=$ iso $>$ ISSN 1726-4634.

13. Elena Andión. Enfermedades en Control de Infecciones. Rev. OMS [Internet]. 2007 octubre 22. [citado 08 Sep 2011]. Disponible en: http://www. ramosmejia.org.ar/s/inf/recomend/intrave.html

14. Colegio de Enfermeros del Perú. Normas de Gestión de la calidad del cuidado enfermero. [Internet].2008 junio 25. [citado 19 Jul 2012]. Disponible en: http:// www.cep.org.pe/beta/download/NG_CDCE.pdf.
15. Mandell D. Enfermedades Infecciones. Principios y Prácticas. 5ta. ed. Buenos Aires: Médica Panamericana; 2007.

16. Barrera M y Cieza M. Factores de riesgo extrínsecos asociados a flebitis en pacientes con vía intravenosa periférica [tesis]. Perú: Universidad Peruana Cayetano Heredia; 2003.

17. Villalonga E. Mesa G. Pérez G, Sandoval S. Llerena F. Cumplimiento de normas técnicas del lavado de manos en áreas de riesgo. Rev Panam Infectol. 2010 noviembre; $12(1): 31-36$.

18. Murray P, Rosenthal K y Pfoller M. Microbiología Médica. 6. ${ }^{a}$ ed. España: El Servier; 2009.

19. Calderdale and HuddersfieldNHS. Aseptic Technique. [Internet].2010 septiembre 18. [citado 16 Feb 2012]. Disponible en: http://www.cht.nhs.uk/fileadmin/ departments/infection_control/policies/Section_G__Aseptic_Technique_Issue_2.pdf.

20. Lenin Humberto Bustamante Ojeda. Evaluación del cumplimiento de las normas de bioseguridad en el Hospital UTPL, en las áreas de emergencia, hospitalización, quirófano, laboratorio y consulta externa, durante el período enero - marzo de 2012 [tesis]. Ecuador. Universidad técnica particular de Loja; 2012.

21. Ponce G., Hernández R. Martínez D. Revista Enfermería Universitaria. 2008 septiembre; 5(5).

22. Becerra N, Calojero E. Aplicación de las Normas de Bioseguridad de los Profesionales de enfermería. [tesis].Venezuela: Universidad De Oriente Núcleo Bolívar; 2010.

23. Casanova L, Castañón J. Reflexiones acerca del lavado de manos. [Internet].2004 mayo 5. [citado 15 agosto 2012] , Rev. Med. IMSS; Vol 42- N. ${ }^{\circ}$ 6519524. Disponible en: http://www.cepis.org.peb vsacdcd30manos2.pdf. Consultado agosto15, 2012.

24. Rosenthal K. Procedimientos intravenosos. Nursing. 2007 abril; 18 (8): 47.

25. Hernán A., William M. Enfermedades Infecciones. Fundamentos de Medicina. 6.a ed. Medellín: Corporación para investigaciones biológicas; 2003.

26. Ministerio de Salud. Manual de Prevención y Control de Infecciones Intrahospitalarias. Oficina General de Epidemiología. Lima; 2008.

27. Organización Mundial de la Salud. Investigación en la Seguridad del paciente.[Internet].2008 marzo 10 [citado 16 Ago 2012].Disponible en: http:// www.who.int/patientsafety/information_centre/ documents/ps_research_brochure_es.pdf

28. Segovia M. Vizcaya N. Cuidados que brinda el profesional de enfermería de atención directa en la 
preparación y administración de medicamentos por vía intravenoso en el centro médico guajás del estado Carabobo durante el primer trimestre del año 2006. [tesis]. Venezuela: Universidad Central de Venezuela; 2007.

29. Alayza L. et al. Factores de riesgo relacionados con la flebitis en pacientes con endovenoso. Medicina y Cirugía. Clínica Ricardo Palma. [tesis].Perú: Universidad Peruana Cayetano Heredia; 2000.

30. Rojas IA. Epidemiología de las Infecciones Intrahospitalarias por Pseudomona aerugionosa en el Hospital Nacional Arzobispo Loayza entre abril y julio de 2002. [tesis].Perú: Universidad Peruana Cayetano Heredia; 2003.

31. Lebeque Y, Moris M, Calas N. Infecciones nosocomiales incidencia de Pseudomona aeruginosa. Rev. Cubana Medicina. 2006 abril; 45(1).

32. Harrison. Principios de Medicina Interna. 18. ${ }^{\mathrm{a}}$ ed. Chile: Mc. Graw-Hill Interamericana; 2005.

33. López S. Medidas de Bioseguridad que utiliza el Personal de Enfermería en el manejo de aspiración de secreciones a pacientes traqueostomizados del Servicio de Cirugía del Instituto Nacional de Enfermedades Neoplásicas. [tesis]. Perú: Universidad Peruana Cayetano Heredia; 2007.

34. Hayden MK, BlomDW, Lyle EA, Moore CG, Weinstein RA. La importancia del lavado de manos y de utilizar guantes para evitar las infecciones cruzadas. Enferm Clin. 2008 agosto; 18(4): 220-2
35. Smeltzer y S. Bare B. Enfermería Medico Quirúrgica de Brunner y Suddarth. 10. ${ }^{\mathrm{a}}$ ed. México D.F: Mc. Graw Hill; 2005. VolII.

36. García M, Mamani I. Infección nosocomillos en pacientes intubados durante el manejo de aspiración de secreciones oro traqueales por enfermería en Hospital de Clínicas e Instituto Nacional de Tórax [tesis]. Bolivia: Universidad Mayor de San Andrés; 2008.

37. Cubas G. Medidas de bioseguridad que aplica el profesional de enfermería en la aspiración de secreciones a pacientes traqueostomizados del Servicio de Emergencia, Hospital Nacional Daniel Alcides Carrión.[tesis]. Perú: Universidad Nacional Mayor de San Marcos; 2009.

\section{Correspondencia}

Esther Chávez Cachay

4848068-987901812

Av. Honorio Delgado 430,

Urb. Ingeniería, S.M.P. Lima - Perú

Correo electrónico: esther.chavez@upch.pe

Forma de citar este artículo: Orellana-Carmona M, ChangaMurga R, Sullcapuma-Guillen B, Chávez Cachay E. Técnicas asépticas en el cuidado enfermero a pacientes hospitalizados que requieren administración de fármacos y aspiración de secreciones del tracto respiratorio. Rev enferm Herediana. 2013;6(2):86-95. 